\section{The role of orbital ultrasonography in distinguishing papilledema from pseudopapilledema}

SB Carter ${ }^{1}$, M Pistilli ${ }^{2}$, KG Livingston ${ }^{3}$, DR Gold ${ }^{3}$, NJ Volpe ${ }^{4}$, KS Shindler ${ }^{3}$, GT Liu ${ }^{5}$ and MA Tamhankar ${ }^{3}$

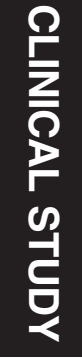

'Department of

Ophthalmology, New York University School of Medicine, New York, NY, USA

management, whereas patients with pseudopapilledema often only need reassurance and/or monitoring. ${ }^{1,2}$ The distinction between these two entities is therefore important in order to avoid potentially invasive and costly testing.

Although clinical history and careful ophthalmoscopic examination are paramount in distinguishing papilledema and pseudopapilledema, ${ }^{1}$ sometimes this distinction can be difficult to make when the patient's history or optic nerve appearance is nondiagnostic. Often patients who have true papilledema may present with minimal or no symptoms, whereas those with pseudopapilledema may complain of unrelated headaches. In such patients, ancillary testing can help to corroborate the clinical impression. These tests include magnetic resonance imaging (MRI) of the brain and orbits to look for structural intracranial lesions or signs of intracranial hypertension; ${ }^{3}$ orbital ultrasonography to look for buried drusen or presence or absence of dilated retrobulbar optic nerve sheaths; ${ }^{4}$ and lumbar puncture to measure intracranial pressure. ${ }^{5}$ In addition, computed tomography (CT) scan of the orbits, ${ }^{6}$ fluorescein angiography, ${ }^{7}$ and optical coherence tomography $(\mathrm{OCT})^{8-10}$ have also been utilized in differentiating these two conditions. Among these modalities, orbital ultrasonography is a useful tool that is rapid, minimally invasive, cost-effective, and poses minimal risk to patient safety. ${ }^{4}$ Previous studies have shown high levels of correlation between optic nerve sheath width by ultrasonography and intracranial pressure measured directly through invasive monitors. ${ }^{11-13}$

A study by Neudorfer et al ${ }^{14}$ showed that increased optic nerve sheath width by orbital ultrasonography correlated well with the final diagnosis of papilledema or pseudopapilledema
${ }^{2}$ Center for Preventive Ophthalmology and Biostatistics, University of Pennsylvania, Philadelphia, PA, USA

${ }^{3}$ Division of NeuroOphthalmology, Scheie Eye Institute, University of Pennsylvania, Philadelphia, PA, USA

${ }^{4}$ Department of Ophthalmology, Feinberg School of Medicine, Northwestern University, Chicago, IL, USA

${ }^{5}$ Division of NeuroOphthalmology, Hospital of University of Pennsylvania, Philadelphia, PA, USA

Correspondence: MA Tamhankar, Division of Neuro-Ophthalmology, Scheie Eye Institute, University of Pennsylvania, $51 \mathrm{~N}$ 39th Street, Philadelphia, PA 19104, USA

Tel: +1 215662 8042;

Fax: +1 2152434694

E-mail: madhura.

tamhankar@uphs.upenn.edu

Received: 20 April 2014 Accepted in revised form: 19 July 2014

Published online:

5 September 2014

Presented in part at the 39th Annual NANOS Meeting, Snowbird, UT, USA,

February 2013 critical distinction to make. Clinical suspicion of papilledema warrants timely evaluation and 
in a pediatric population. Our study aims to further evaluate the role of orbital ultrasonography in distinguishing papilledema from pseudopapilledema in a cohort of adult patients who were referred to the neuroophthalmology service for the evaluation of swollen optic nerves. Moreover, we utilized baseline fundus photography with subsequent documentation of change or no change in the optic nerve appearance by the examining neuro-ophthalmologist as an additional objective parameter against which the ultrasonography results were compared.

\section{Materials and methods}

A retrospective chart review was conducted of all adults who were referred to the neuro-ophthalmology service over a 14-year period and underwent orbital ultrasonography and fundus photography as part of their evaluation for papilledema. Orbital ultrasonography was performed by a single experienced ultrasonographer. In general, our cohort of patients represented those with mild disc swelling or with an ambiguous diagnosis by history and clinical exam, whereas those with more obvious disc swelling with hemorrhages and/or exudates did not usually undergo this testing. All enrolled patients were required to have at least two neuro-ophthalmologic visits that were $\geq 30$ days apart in addition to photographic documentation of the optic nerve head appearance during the initial presentation. The enrollment criteria also included specific documentation of subsequent change or no change in optic nerve appearance as commented by the neuro-ophthalmologist in the follow-up notes. Patients with prior intracranial or orbital surgeries were excluded from the study. Papilledema was defined as optic nerve head swelling due to raised intracranial pressure, ${ }^{15,16}$ whereas pseudopapilledema was defined as optic nerve head elevation present in conjunction with anomalous optic nerves and/or buried optic nerve head drusen. ${ }^{17}$ Patients with insufficient follow-up time, lack of fundus photography, and aged $<18$ years were excluded from the study. The study design was approved by the University of Pennsylvania institutional review board.

Orbital ultrasonography was performed using an Ellex Eye Cubed A/B System (Ellex Medical Pty Ltd, Adelaide, SA, Australia) equipped with a $10-\mathrm{MHz}$ transducer in A- and B-scan modes. B-scan mode was used to provide a two-dimensional cross-sectional image of the eye to identify optic disc elevation and look for a fluid (crescent) sign. A-scan mode, which is standardized for tissue differentiation with two gates for measurement, was used to determine the optic nerve sheath width in the primary gaze. If the optic nerve sheath was found to be wide $(\geq 3.3 \mathrm{~mm}),{ }^{14,18}$ a $30^{\circ}$ test was then performed to differentiate fluid vs solid thickening of the optic nerve sheath. ${ }^{4}$ The patient was instructed to re-fixate at least $30^{\circ}$ away from the primary gaze toward the ultrasound probe, at which point the optic nerve sheath was measured again. If fluid is present, the measured width of the optic nerve is expected to decrease, based on the assumption that when the eye is turned the optic nerve sheaths become stretched and the fluid is redistributed over a greater area. A reduction in width $>10 \%$ was considered positive for the presence of fluid. ${ }^{4}$

Ultrasonographic findings that were recorded included optic nerve sheath width and presence or absence of a $30^{\circ}$ sign. A positive result by ultrasonography was defined as both an optic nerve sheath width $\geq 3.3 \mathrm{~mm}$ and a positive $30^{\circ}$ sign. In addition, details of the patient's medical history, neuroophthalmic examination, and results of ancillary testing including MRI and lumbar puncture were obtained. An opening pressure of $\geq 25 \mathrm{~cm} \mathrm{H}_{2} \mathrm{O}$ on lumbar puncture was considered abnormally elevated. The findings of orbital ultrasonography were then correlated with the final diagnosis of papilledema or pseudopapilledema as determined by the neuro-ophthalmologist based on clinical impression.

All statistical analyses were performed with SAS version 9.3 (SAS Corporation, Cary, NC, USA). Comparisons between the papilledema and pseudopapilledema patients were made using a $t$-test for age, Wilcoxon rank-sum test for duration of follow-up, and chi-square tests for all categorical characteristics. Sensitivity and specificity were calculated to assess the ability of orbital ultrasonography and lumbar puncture to discriminate papilledema from pseudopapilledema based on the final diagnosis as ascertained by the neuro-ophthalmologist and are reported with $95 \%$ confidence intervals.

\section{Results}

Of the 407 patients who underwent orbital ultrasonography for the evaluation of suspected papilledema, 87 patients met the inclusion criteria and were enrolled in the study. The majority of those excluded lacked sufficient follow-up time or baseline fundus photography. The average age of the study cohort was 39 years $(S D \pm 13.8)$. A comparison of patient characteristics between the papilledema and pseudopapilledema groups is presented in Table 1. The mean age of patients with pseudopapilledema was higher than those with papilledema, whereas the gender distribution was similar in both the groups. The median time of follow-up for all patients was 7 months (range $=1-85$ months), with a longer follow-up for patients with papilledema. 
Table 1 Patient characteristics

\begin{tabular}{|c|c|c|c|c|}
\hline & All $(\mathrm{n}=87)$ & $\begin{array}{c}\text { Papilledema } \\
(\mathrm{n}=39,44.8 \%)\end{array}$ & $\begin{array}{l}\text { Pseudopapilledema } \\
\quad(\mathrm{n}=48,55.2 \%)\end{array}$ & P-value ${ }^{\mathrm{a}}$ \\
\hline Mean (SD) age in years & $39.0(13.8)$ & $35.2(12.2)$ & $42.0(14.4)$ & 0.02 \\
\hline Female (\%) & $69(79.3 \%)$ & $31(79.5 \%)$ & $38(79.2 \%)$ & 0.97 \\
\hline Median (min - max) months of follow-up & $7(1-85)$ & $14(1-81)$ & $6(1-85)$ & $<0.01$ \\
\hline History of weight gain (\%) & $26(29.9 \%)$ & $21(53.9 \%)$ & $5(10.4 \%)$ & $<0.001$ \\
\hline Overweight (\%) & $60(69.0 \%)$ & $36(92.3 \%)$ & $24(50.0 \%)$ & $<0.001$ \\
\hline \multicolumn{5}{|l|}{ Chief complaint (\%) } \\
\hline Asymptomatic & $41(47.1 \%)$ & $9(23.1 \%)$ & $32(66.7 \%)$ & $<0.001$ \\
\hline Headaches & $20(23.0 \%)$ & $17(43.6 \%)$ & $3(6.3 \%)$ & \\
\hline Transient visual obscurations & $9(10.3 \%)$ & $8(20.5 \%)$ & $1(2.1 \%)$ & \\
\hline Blurry vision & $10(11.5 \%)$ & $0(0 \%)$ & $10(20.8 \%)$ & \\
\hline Others & $7(8.1 \%)$ & $5(12.8 \%)$ & $2(4.2 \%)$ & \\
\hline
\end{tabular}

${ }^{a} P$-values calculated using $t$-test for age, Wilcoxon rank-sum test for months of follow-up, and chi-square test for all others.

Of the cohort, $45 \%(39 / 87)$ of patients were diagnosed with papilledema and 55\% (48/87) were diagnosed with pseudopapilledema. As indicated in Table 1, there were significant differences between the papilledema and pseudopapilledema patients with respect to their symptoms $(P<0.001)$. The majority of patients who had pseudopapilledema were asymptomatic $(67 \%)$ or presented with blurry vision $(21 \%)$, whereas $77 \%$ of patients with papilledema had symptoms. The most common complaints among patients with papilledema were headache $(44 \%)$ and transient visual obscurations (21\%). A higher proportion of patients with papilledema were overweight (body mass index (BMI) $\geq 25,92 \%$ vs $50 \%$ ), and were more likely to report a history of weight gain $(54 \%$ vs $10 \%)$.

The sensitivity of orbital ultrasonography in diagnosing papilledema was $90 \%$ ( $n=35 / 39$, CI: 80.2 $99.3 \%$ ). Of the four patients who were diagnosed with papilledema (three females and one male) and had normal ultrasonography (false negatives), all four were symptomatic with mild optic nerve swelling, absence of hemorrhages and/or exudates, good visual function, and a documented change in their optic nerve head appearance over time (average follow-up of 6 months). The average optic nerve sheath width in these four patients was $2.7 \mathrm{~mm}$, which is the same as that for patients with pseudopapilledema $(2.7 \mathrm{~mm})$. Three of the four patients had classic symptoms of raised intracranial pressure (headaches and/or transient visual obscurations). In addition, three of the four patients had elevated opening pressures on diagnostic lumbar puncture and one patient had MRI signs of raised intracranial pressure (flattening of the posterior globes).

In the group of patients with pseudopapilledema, the specificity of orbital ultrasonography was $79 \%$ ( $n=38 / 48$, CI: $67.7-90.7 \%$ ). Of the $21 \%$ of patients $(10 / 48)$, who were diagnosed with pseudopapilledema and had positive ultrasonography (false positives), 9 of the 10 patients were females who were asymptomatic and 1 patient complained of blurry vision that was found to be refractive in nature. None of the 10 patients had hemorrhages, exudates, or vessel obscurations in association with elevated optic nerve heads. Six of the 10 patients had anomalous disc vasculature, a characteristic of pseudopapilledema. ${ }^{14}$ None of the patients showed any change in optic nerve appearance over time (average follow-up of 10 months). The average optic nerve sheath width by ultrasonography in these patients was $4.7 \mathrm{~mm}$, which is the same as that for patients with papilledema $(4.8 \mathrm{~mm})$.

Lumbar puncture was found to be $90 \%$ sensitive for diagnosis of papilledema $(n=27 / 30, \mathrm{CI}=79.3-100 \%)$. Of the three patients with papilledema and negative lumbar punctures (opening pressure $\leq 25 \mathrm{~cm}$ of $\mathrm{H}_{2} \mathrm{O}$ ), two were on acetazolamide at the time of their lumbar punctures and were found to have opening pressures of 18 and $20 \mathrm{~cm}$ of $\mathrm{H}_{2} \mathrm{O}$, and the third patient had a borderline opening pressure of $24 \mathrm{~cm}$ of $\mathrm{H}_{2} \mathrm{O}$ with documented change in optic nerve appearance. All three patients had positive ultrasonography. The specificity of lumbar puncture for pseudopapilledema was $67 \%$ for the three patients who underwent the procedure $(n=2 / 3$, $\mathrm{CI}=13.4-100 \%)$. The one patient who was diagnosed with pseudopapilledema and had a positive lumbar puncture was asymptomatic with a normal MRI and a borderline opening pressure of $25 \mathrm{~cm}$ of $\mathrm{H}_{2} \mathrm{O}$.

\section{Discussion}

Our results indicate that orbital ultrasonography is a highly sensitive (90\%) but less specific tool $(79 \%)$ in the detection of papilledema in a subset of patients referred to the neuro-ophthalmologic service for optic disc swelling. In patients with atypical optic nerve elevation, 
orbital ultrasonography is a valuable ancillary test for guiding further management.

Our results compare favorably with a previous investigation looking at the utility of orbital ultrasonography in distinguishing papilledema from pseudopapilledema by Neudorfer et al, ${ }^{14}$ who found a sensitivity of $85 \%$ and specificity of $63 \%$ when the upper limit of normal optic nerve sheath width was set at the same level as our study $(3.3 \mathrm{~mm})$. In comparison, we found a slightly higher level of sensitivity (90\%) and specificity (79\%) in our study, which was larger (87 vs 44 patients) and included only adults rather than children (average age 39 vs 12.7 years). The higher sensitivity found in our study may also be due to the requirement of two positive ultrasound parameters (optic nerve sheath width $\geq 3.3 \mathrm{~mm}$ and a $30^{\circ}$ sign), compared with the Neudorfer study, which used only optic nerve sheath width. Our study also used fundus photographs with documentation of change or no change in the optic disc appearance as an additional objective measure to differentiate papilledema from pseudopapilledema.

The inclusion requirements of our study of fundus photography and significant neuro-ophthalmologic follow-up are particularly important, as in the absence of a gold standard test for papilledema, change in the optic nerve appearance over time may be the only objective indicator to guide the clinician to the correct diagnosis in some patients with ambiguous optic disc appearance.

The finding of normal optic nerve sheath width seen in $10 \%$ of our cohort with papilledema underscores the importance of considering clinical signs and symptoms in establishing a diagnosis of papilledema. The four patients diagnosed with papilledema who had normal optic nerve sheath widths were all symptomatic, and three of them had elevated opening pressures on lumbar puncture. It is possible that the threshold for optic nerve swelling in these patients may be lower, and that optic nerve swelling may occur with 'normal' optic nerve sheath diameters in a small subset of patients. The average optic nerve sheath diameter in these patients was identical to the normal optic nerve sheath widths seen in patients with pseudopapilledema, suggesting that lowering the cutoff for optic nerve sheath width would not aid in identifying them. In such patients, if symptoms of raised intracranial pressure are present, further testing should be considered.

Orbital ultrasonography was less specific in the diagnosis of pseudopapilledema (79\%), with $21 \%$ of patients found to have dilated optic nerve sheaths. This finding suggests that in patients who do not have a history suggestive of intracranial hypertension but have swollen optic nerves, clinicians should interpret positive ultrasonography cautiously. In such patients, lack of symptoms and presence of anomalous vascular anatomy on the surface of the optic nerves may be a helpful clue in making the diagnosis of pseudopapilledema. ${ }^{19}$ In these 10 patients who had dilated optic nerve sheath widths, 3 underwent lumbar puncture and 1 of those 3 had a borderline elevated opening pressure. There was no lumbar puncture performed in the remaining seven patients. Although it could be argued that the one patient with opening pressure of $25 \mathrm{~cm}$ of $\mathrm{H}_{2} \mathrm{O}$ and some of the remaining seven may have had true papilledema, the absence of symptoms of raised intracranial pressure and no change in disc appearance over time makes true papilledema unlikely. Moreover, 3 of the 4 patients with negative ultrasound were favored to have papilledema and 9 of the 10 patients with positive ultrasound were clinically felt to have pseudopapilledema by the examining neuro-ophthalmologist. This underscores the importance of having a high clinical suspicion before ordering ancillary tests.

In support of patients who have pseudopapilledema and dilated optic nerve sheaths, there is one case report describing a patient with bilateral optic disc elevation and several positive $30^{\circ}$ tests by ultrasonography, suggesting increased level of subarachnoid fluid in the optic nerve sheath. ${ }^{20}$ The patient remained symptomatic over an 8-year period and had three lumbar punctures with normal opening pressures without any change in optic disc appearance. The authors suggest that patients may have variable optic nerve sheath compliance, with some patients reaching dilated widths even with normal intracranial pressure, or that the nerve sheaths may simply be patulous. From our study it appears that within the subset of patients with indeterminate optic nerve appearance, this phenomenon may be more common than previously appreciated. Such patulous optic nerve sheaths are also occasionally noted on MRI scans performed on patients without elevated intracranial pressure, suggesting that this can be a normal variation.

Our study results indicate that the sensitivity of lumbar puncture was similar to orbital ultrasonography in the detection of papilledema $(90 \%)$, although it was less specific (67\%). These values may have been confounded by the fact that lumbar puncture was often obtained only after a significant suspicion of true papilledema was established, and was therefore not ordered when the patients were thought to have pseudopapilledema. This may explain why only three patients in our study with pseudopapilledema had lumbar punctures performed. Although spinal fluid analysis is indicated to rule out other etiologies for raised intracranial pressure, opening pressure measurements can potentially vary in some patients based on many factors such as patient positioning with prone position recordings being higher than lateral decubitus, ${ }^{21,22}$ 
Valsalva maneuvers, ${ }^{23}$ effect of anesthesia, ${ }^{24}$ and cannot be solely relied upon in making an accurate diagnosis. In our study, there was one patient who had CSF opening pressure measurement of $24 \mathrm{~cm}$ of $\mathrm{H}_{2} \mathrm{O}$ who had normal ultrasound and clinical features suspicious for papilledema and one patient in the pseudopapilledema category with $25 \mathrm{~cm} \mathrm{H}_{2} \mathrm{O}$ of opening pressure. This underscores the importance of having a level of suspicion before ordering invasive testing that also may give variable results.

Although our study was not powered to assess the overall sensitivity and specificity of MRI signs of raised intracranial pressure in distinguishing papilledema from pseudopapilledema, our results suggest that orbital ultrasonography compares favorably with previous studies that have investigated the role of MRI in the diagnosis of idiopathic intracranial hypertension (IIH). ${ }^{25,26}$ In a recent study of patients with $\mathrm{IIH}$, it was found that increased optic nerve sheath diameter identified on MRI scan was $72-80 \%$ sensitive and $96 \%$ specific for intracranial hypertension. ${ }^{26}$ The only other reliable sign was shortening of the pituitary gland that was $88 \%$ sensitive and $76 \%$ specific. To our knowledge, no study to date has sufficiently correlated these MRI findings with the presence or absence of papilledema or evaluated the frequency and accuracy of reporting these radiographic signs in everyday practice.

The limitations of our study include a retrospective collection of data and selection bias. The subset of patients with suspected optic nerve head edema who were referred for ultrasonography may preferentially represent those for whom the presentation and optic nerve appearance by ophthalmoscopy was more equivocal. Nevertheless, this would skew our study toward those patients in whom there was a diagnostic dilemma regarding the etiology of the optic nerve swelling, and therefore represents a particularly important cohort of patients in whom the management may be altered on the basis of the findings of orbital ultrasonography. Another limitation of this procedure is the ready availability of a trained ultrasonographer who can read A and B scans. It is currently estimated that there are $>150$ dedicated orbital ultrasonographers in academic centers across the United States and for some physicians interested in ordering these tests, lack of access to these professionals may limit its usage. It is also important to realize that the threshold for cutoff for normal optic nerve sheath diameters depends on the frequency of the probe used and different normative data exist based on the linear transducer frequency and whether the measurements are taken using A or B scan.

The majority of the patients in our study were managed in an era before spectral domain OCT, which may also prove to be an important ancillary test in this differential diagnosis. ${ }^{8-10}$ Nevertheless, OCT is limited by its inability to examine the retrobulbar anatomy or identify optic nerve sheath abnormalities, which are important advantages to orbital ultrasonography. ${ }^{27}$ A recent study failed to find any significant difference in retinal nerve fiber layer thickness between patients with buried optic nerve head drusen and those with mild papilledema secondary to IIH using spectral domain OCT. ${ }^{28}$ The reported diagnostic accuracy among trained readers of these images was also poor, suggesting that ultrasonography may be both quantitatively and qualitatively more suitable for this application.

Many practitioners also rely on intravenous fluorescein angiographic staining patterns to distinguish true optic disc edema from buried optic disc drusen. ${ }^{29}$ However, the fluorescein angiographic findings can be misinterpreted, especially when differentiating between dye leakage and staining. This is particularly true in persons who have pseudopapilledema without drusen (false-positive patients in our cohort).

Although there is no ancillary test that can replace an accurate history and examination to distinguish between papilledema and pseudopapilledema, our study shows that orbital ultrasonography serves as a valuable adjunct to clinical diagnosis. Asymptomatic patients who present with optic disc swelling and normal optic nerve sheath width may be followed with serial photographs to look for changes in appearance, sparing them the time and unnecessary expense of MRI and lumbar puncture, whereas patients who present with symptoms of raised intracranial pressure should have further testing done despite negative ultrasonographic results.

\section{Summary}

\section{What was known before}

- Orbital ultrasonography is a useful and minimally invasive tool that helps to differentiate between papilledema and pseudopapilledema. Clinical suspicion of papilledema warrants timely evaluation and management, whereas patients with pseudopapilledema often only need reassurance and/or monitoring. The distinction between these two entities is therefore important in order to avoid potentially invasive and costly testing.

- The sensitivity and specificity of orbital ultrasonography in distinguishing papilledema from pseudopapilledema is not well studied in adults.

\section{What this study adds}

- Although clinical history and careful ophthalmoscopic examination are paramount in distinguishing between papilledema and pseudopapilledema, our study demonstrates that orbital ultrasonography is a highly sensitive but less specific tool that is useful in guiding further management in patients for whom the diagnosis is initially uncertain. 


\section{Conflict of interest}

The authors declare no conflict of interest.

\section{References}

1 Savino PJ, Glaser JS. Pseudopapilledema versus papilledema. Int Ophthal Clin 1977; 17: 115-137.

2 Hoyt WF, Pont ME. Pseudopapilledema: anomalous elevation of optic disk: pitfalls in diagnosis and management. JAMA 1962; 181: 191-196.

3 Agid R, Farb RI, Willinsky RA, Mikulis DJ, Tomlinson G. Idiopathic intracranial hypertension: the validity of cross-sectional neuroimaging signs. Neuroradiology 2006; 48: 521-527.

4 Atta HR. Imaging of the optic nerve with standardised echography. Eye (Lond) 1988; 2: 358-366.

5 Biousse V, Bruce BB, Newman NJ. Update on the pathophysiology and management of idiopathic intracranial hypertension. J Neurol Neurosurg Psychiatry 2012; 83: 488-494.

6 Kurz-Levin M, Landau K. A comparison of imaging techniques for diagnosing drusen of the optic nerve head. Arch Ophthalmol 1999; 117: 1045-1049.

7 Pineles SL, Arnold AC. Fluorescein angiographic identification of optic disc drusen with and without optic disc edema. J Neuroophthalmol 2012; 32: 17-22.

8 Sarac O, Tasci YY, Gurdal C, Can I. Differentiation of optic disc edema from optic nerve head drusen with spectral-domain optical coherence tomography. J Neuroophthalmol 2012; 32: 207-211.

9 Lee KM, Woo SJ, Hwang JM. Differentiation of optic nerve head drusen and optic disc edema with spectral-domain optical coherence tomography. Ophthalmology 2011; 118: 971-977.

10 Flores-Rodríguez P, Gili P, Martín-Ríos MD. Sensitivity and specificity of time-domain and spectral-domain optical coherence tomography in differentiating optic nerve head drusen and optic disc oedema. Ophthalmic Physiol Opt 2012; 32: 213-221.

11 Rajajee V, Vanaman M, Fletcher JJ, Jacobs TL. Optic nerve ultrasound for the detection of raised intracranial pressure. Neurocrit Care 2011; 15: 506-515.

12 Kimberly HH, Shah S, Marill K, Noble V. Correlation of optic nerve sheath diameter with direct measurement of intracranial pressure. Acad Emerg Med 2008; 15: 201-204.

13 Geeraerts T, Launey Y, Martin L, Pottecher J, Vigue B, Duranteau J et al. Ultrasonography of the optic nerve sheath may be useful for detecting raised intracranial pressure after severe brain injury. Intensive Care Med 2007; 33: 1704-1711.
14 Neudorfer M, Ben-Haim MS, Leibovitch I, Kesler A. The efficacy of optic nerve ultrasonography for differentiating papilloedema from pseudopapilloedema in eyes with swollen optic discs. Acta Ophthalmol 2013; 91(4): 376-380.

15 Minckler DS, Tso MO, Zimmerman LE. A light microscopic, autoradiographic study of axoplasmic transport in the optic nerve head during ocular hypotony, increased intraocular pressure, and papilledema. Am J Ophthalmol 1976; 82: 741-757.

16 Tso MO, Hayreh SS. Optic disc edema in raised intracranial pressure. Arch Ophthalmol 1977; 95: 1458-1462.

17 Davis PL, Jay WM. Optic nerve head drusen. Semin Ophthalmol 2003; 18: 222-242.

18 Byrne SF, Green RL. Ultrasound of the Eye and Orbit. 2nd edn. Mosby: St. Louis, 2002.

19 Rosenberg MA, Savino PJ, Glaser JS. A clinical analysis of pseudopapilledema. Arch Ophthalmol 1979; 97: 65-70.

20 Sadda SR, DiBernardo C, Miller NR. Anomalous optic disc elevation associated with ultrasonographic evidence of increased subarachnoid fluid. J Neuroophthalmol 2000; 20: 25-27.

21 Abbrescia KL, Brabson TA, Dalsey WC, Kelly JJ, Kaplan JL, Young TM et al. The effect of lower-extremity position on cerebrospinal fluid pressures. Acad Emerg Med 2001; 8: 8-12.

22 Schwartz KM, Luetmer PH, Hunt $\mathrm{CH}$, Kotsenas $\mathrm{AL}$, Diehn FE, Eckel LJ et al. Position-related variability of CSF opening pressure measurements. AJNR Am J Neuroradiol 2012; 34: 904-907.

23 Neville L, Egan RA. Frequency and amplitude of elevation of cerebrospinal fluid resting pressure by the Valsalva maneuver. Can J Ophthalmol 2005; 40: 775-777.

24 Eidlitz-Markus T, Stiebel-Kalish H, Rubin Y, Shuper A. CSF pressure measurement during anesthesia: an unreliable technique. Paediatr Anaesth 2005; 15(12): 1078-1082.

25 Brodsky MC, Vaphiades M. Magnetic resonance imaging in pseudotumor cerebri. Ophthalmology 1998; 105: 1686-1693.

26 Hoffmann J, Huppertz HJ, Schmidt C, Kunte H, Harms L, Klingebiel $\mathrm{R}$ et al. Morphometric and volumetric MRI changes in idiopathic intracranial hypertension. Cephalalgia 2013; 33: 1075-1084.

27 Sakata LM, Deleon-Ortega J, Sakata V, Girkin CA. Optical coherence tomography of the retina and optic nerve - a review. Clin Experiment Ophthalmol 2009; 37: 90-99.

28 Kulkarni KM, Pasol J, Rosa PR, Lam BL. Differentiating mild papilledema and buried optic nerve head drusen using spectral domain optical coherence tomography. Ophthalmology 2014; 121: 959-963.

29 Pineles SL, Arnold AC. Fluorescein angiographic identification of optic disc drusen with and without optic disc edema. J Neuroophthalmol 2012; 32: 17-22. 